\title{
Lattice disorder and oxygen migration pathways in pyrochlore and defect-fluorite oxides
}

\author{
Frederick P. Marlton ${ }^{1}$, Zhaoming Zhang ${ }^{2}$, Yuanpeng Zhang ${ }^{3}$, Thomas E. Proffen ${ }^{3}$, Chris D. Ling ${ }^{1}$, \\ Brendan J. Kennedy ${ }^{1}$ \\ ${ }^{1}$ School of Chemistry, The University of Sydney, Sydney, NSW 2006, Australia, \\ ${ }^{2}$ Australian Nuclear Science and Technology Organisation, Lucas Heights, NSW 2234, Australia, \\ ${ }^{3}$ Neutron Scattering Division, Oak Ridge National Laboratory, Oak Ridge, TN 37831, USA
}

Frederick.marlton@sydney.edu.au

Pyrochlore oxides, with the general formula $A_{2} B_{2} \mathrm{O}_{7}$, are of considerable interest as catalysts for the oxygen evolution reaction[1-5], where $A_{2} \mathrm{Ru}_{2} \mathrm{O}_{7-\delta}$ pyrochlores have recently emerged as state-of-the-art materials, and as photocatalysts for hydrogen evolution[6-8]. Fundamental to their reactivity is the local-scale vacancy ordering and mobility, which can be tailored through cation substitution[4]. The chemical and structural flexibility of pyrochlore oxides gives them a diverse range of physical and chemical properties leading to technological applications including as fast-ion conductors[9, 10], ferroelectrics[11], magnetism[12], oxide heterostructures[13, 14], and host matrices for the immobilization of actinide-rich nuclear wastes[15].

Atomic-scale disorder plays an important role in the chemical and physical properties of oxide materials. The structural flexibility of pyrochlore-type oxides allows for crystal-chemical engineering of these properties. Compositional modification can push pyrochlore oxides towards a disordered defect-fluorite structure with anion Frenkel pair defects that facilitate oxygen migration. The local structure of the long-range average cubic defect-fluorite was recently claimed to consist of randomly arranged orthorhombic weberitetype domains[16]. In this work we show, using low-temperature neutron total-scattering experiments, that this is not the case for $\mathrm{Zr}$ rich defect-fluorites. By analyzing data from the pyrochlore/defect-fluorite $\mathrm{Y}_{2} \mathrm{Sn}_{2-x} \mathrm{Zr}_{x} \mathrm{O}_{7}$ series using a combination of neutron pair distribution function and big-box modelling, we have differentiated and quantified the relationship between anion sub-lattice disorder and Frenkel defects. These details directly influence the energy landscape for oxygen migration and are crucial for simulations and design of new materials with improved properties.

[1] Shang, C., et al., Electron Correlations Engineer Catalytic Activity of Pyrochlore Iridates for Acidic Water Oxidation. Advanced Materials, 2019. 31(6): p. 1805104.

[2] Oh, S.H., et al., Synthesis of a metallic mesoporous pyrochlore as a catalyst for lithium-O2 batteries. Nature Chemistry, 2012. 4(12): p. 10041010 .

[3] Cheng, F. and J. Chen, Something from nothing. Nature Chemistry, 2012. 4(12): p. 962-963.

[4] Kuznetsov, D.A., et al., Tailoring Lattice Oxygen Binding in Ruthenium Pyrochlores to Enhance Oxygen Evolution Activity. Journal of the American Chemical Society, 2020. 142(17): p. 7883-7888.

[5] Abbott, D.F., et al., Design and Synthesis of Ir/Ru Pyrochlore Catalysts for the Oxygen Evolution Reaction Based on Their Bulk Thermodynamic Properties. ACS Applied Materials \& Interfaces, 2019. 11(41): p. 37748-37760.

[6] Allured, B., et al., Enhancing the visible light absorbance of Bi2Ti2O7 through Fe-substitution and its effects on photocatalytic hydrogen evolution. Applied Catalysis B: Environmental, 2014. 144: p. 261-268.

[7] Wu, J., et al., One-Pot Hydrothermal Synthesis and Photocatalytic Hydrogen Evolution of Pyrochlore Type K2Nb2O6. Chinese Journal of Chemistry, 2014. 32(6): p. 485-490.

[8] Kuriki, R., et al., A Stable, Narrow-Gap Oxyfluoride Photocatalyst for Visible-Light Hydrogen Evolution and Carbon Dioxide Reduction. Journal of the American Chemical Society, 2018. 140(21): p. 6648-6655.

[9] Radhakrishnan, A.N., et al., Influence of disorder-to-order transition on lattice thermal expansion and oxide ion conductivity in (CaxGd1-x)2(Zr1-xMx)2O7 pyrochlore solid solutions. Dalton Transactions, 2011. 40(15): p. 3839.

[10] Garcia-Barriocanal, J., et al., Tailoring Disorder and Dimensionality: Strategies for Improved Solid Oxide Fuel Cell Electrolytes. ChemPhysChem, 2009. 10(7): p. 1003-1011.

[11] McQueen, T.M., et al., Frustrated ferroelectricity in niobate pyrochlores. Journal of Physics: Condensed Matter, 2008. 20(23): p. 235210. 
[12] Thygesen, P.M.M., et al., Orbital Dimer Model for the Spin-Glass State in Y2Mo2O7. Physical Review Letters, 2017. 118(6).

[13] O'Sullivan, M., et al., Interface control by chemical and dimensional matching in an oxide heterostructure. Nature Chemistry, 2016. 8(4): p. 347353.

[14] Poeppelmeier, K. R.; Rondinelli, J. M., Mismatched lattices patched up. Nature Chemistry 2016, 8 (4), $292-294$.

[15] Ewing, R.C., W.J. Weber, and J. Lian, Nuclear waste disposal—pyrochlore (A2B2O7): Nuclear waste form for the immobilization of plutonium and "minor" actinides. Journal of Applied Physics, 2004. 95(11): p. 5949-5971.

[16] Shamblin, J., et al., Probing disorder in isometric pyrochlore and related complex oxides. Nature Materials, 2016. 15(5): p. 507-511.

Keywords: Pyrochlores; fluorites; disorder; local-structure; total-scattering 